\title{
Adhesive wear mechanisms uncovered by atomistic simulations
}

\author{
Jean-François MOLINARI ${ }^{1,}{ }^{*}$, Ramin AGHABABAEI $^{2}$, Tobias BRINK ${ }^{1}$, Lucas FRÉROT $^{1}$, Enrico MILANESE $^{1}$ \\ ${ }^{1}$ Civil Engineering Department, Materials Science Department, École Polytechnique Fédérale de Lausanne, Lausanne 1015, Switzerland \\ ${ }^{2}$ Department of Engineering - Mechanical Engineering, Aarhus Universitet, Aarhus 8000, Denmark \\ Received: 01 May 2018 / Revised: 14 July 2018 / Accepted: 16 July 2018 \\ (C) The author(s) 2018. This article is published with open access at Springerlink.com
}

\begin{abstract}
In this review, we discuss our recent advances in modeling adhesive wear mechanisms using coarse-grained atomistic simulations. In particular, we present how a model pair potential reveals the transition from ductile shearing of an asperity to the formation of a debris particle. This transition occurs at a critical junction size, which determines the particle size at its birth. Atomistic simulations also reveal that for nearby asperities, crack shielding mechanisms result in a wear volume proportional to an effective area larger than the real contact area. As the density of microcontacts increases with load, we propose this crack shielding mechanism as a key to understand the transition from mild to severe wear. We conclude with open questions and a road map to incorporate these findings in mesoscale continuum models. Because these mesoscale models allow an accurate statistical representation of rough surfaces, they provide a simple means to interpret classical phenomenological wear models and wear coefficients from physics-based principles.
\end{abstract}

Keywords: adhesive wear; molecular dynamics; continuum mechanics

\section{Introduction}

In 1995, when Meng and Ludema [1] reviewed an extensive literature of around 300 equations for friction and wear, times were dire for tribology. Progress had arguably slowed down since the golden age of tribology in the 1950s, and research funding from national science foundations in various countries was being cut for tribology research and reallocated to other scientific fields. This was happening despite the importance of understanding and controlling wear mechanisms for industry and society at large. After a detailed analysis of wear models, including their origin, content and applicability, Meng and Ludema concluded that "No single predictive equation or group of limited equations could be found for general and practical use. The reasons include the perpetuation of erroneous and subjective expressions for the mechanisms of wear, the slow pace of translation of microscopic observations into macroscopic models of the wearing processes and the paucity of good experiments to verify proposed models".

Meng and Ludema's review came after three centuries of scientific investigations on wear mechanisms, which started in 1803 with Hatchett [2]. This had led to the emergence of a myriad of empirical/ phenomenological models, often with increasing complexity (Meng and Ludema, for instance, refer to one model with 26 independent material parameters). Amongst them is the ubiquitous Archard wear law [3], which will be discussed at length in this paper. Archard's law is arguably the simplest existing wear model, and we here briefly recall its proposition: the wear volume (total volume of collected debris) is proportional to the normal load, the sliding distance, and inversely proportional to the hardness of the softer material in contact. Simple theoretical arguments [3]

* Corresponding author: Jean-François MOLINARI, E-mail: jean-francois.molinari@epfl.ch 
can be brought forward to explain the mechanistic origins of this model, but the theoretical prediction does not agree with experimental data without using a proportionality constant, the wear coefficient $K$. This coefficient is essentially an experimental fitting parameter, and it is not a small correction, but a strong adjustment of several orders of magnitude. Typical values for the wear coefficient are between $10^{-2}$ to $10^{-8}$. The origin of $K$ is unclear, but $K$ is usually interpreted as a probability, because only a few contact asperities amongst the many form wear particles. It is telling that even the simplest wear model necessitates an empirical adjustement. As stated by Meng and Ludema, by and large, our macroscopic engineeringscale understanding of wear remains limited $[1,4]$.

However, this does not come as a surprise to the initiated tribologist, because it is hard to find a more complex and dirty problem than wear. After all, wear processes emerge from a variety of physical and chemical mechanisms at disparate time and length scales. Wear comes in many forms, amongst which adhesive and abrasive wear are the most prominent [5]. Wear processes depend on environmental conditions and vary with time. It is impossible to draw up an exhaustive list here. They depend on the rate of physico-chemical reactions, and also on the evolution of surface roughness that dictates the geometry and pressures at microcontacts. In lubricated contacts the lubricant properties may degrade with time. The wear particles and sheared materials form a tribolayer with complex properties and in turn alter the surface properties. All this complexity resulted in little alternatives to empirical models relying on parameters calibrated for specific materials and applications.

A major advancement came in the 1980s/1990s thanks to nanotribology. Atomic-force microscopy (AFM) provided a means to explore the fundamental mechanisms leading to friction and wear. A turn was taken towards identifying molecular mechanisms experimentally and opening a dialogue with increasingly reliable numerical simulations. Numerical modeling of wear processes is appealing as it opens the possibility to zoom in on an otherwise buried contact interface. However, numerical modeling comes with its own difficulties. These stem from the challenge of scales of tribological problems and once more from the complex physico-chemical mechanisms taking place at the contact interface. Let us consider the challenge of scales for a moment, and limit ourselves to the question of relevant length scales for wear processes (the question of relevant time scales is equally if not more important but will be left out of the discussion of this paper). One may resort to atomistic simulations, as for instance with classical molecular dynamics (MD). This is a very useful approach in particular because it is relevant in scale to AFM experimental data [6-11]. However, classical MD simulations are limited to sizes below microns, which are disconnected from the vast category of engineering wear scenarios, in which wear particles are of the order of, or above, micrometers, i.e., orders of magnitude larger than the scale at which MD simulations operate. Alternatively, wear modeling approaches can be conducted at a larger scale, at which one would apply efficient continuum mechanics models. This is for instance the approach taken with the finite-element method (FEM) or with the boundary-element method (BEM). Besides computational efficiency, a major advantage of these numerical approaches is that it is fairly straightforward to account for diverse constitutive behaviors. But a major disadvantage is that they do not give insights on molecular mechanisms, and struggle to handle the large deformations, tearing, breaking and mixing of materials resulting from wear processes.

This challenge of scales has motivated us to work at an intermediate scale, denoted here by mesoscale, and to revisit the classical Archard wear law. The present manuscript reviews and discusses the implications of our recent results. It is organized in two steps: we will first describe our efforts at capturing the atomistic mechanisms leading to the formation of wear debris. This will be conducted using classical molecular dynamics. Then we will incorporate our findings in a mesoscale continuum mechanics BEM solver. The focus of the paper is on adhesive wear, i.e., the formation of debris particles when contact junctions form under strong adhesive bonds. We restrict our attention to dry sliding between two identical solid materials. We will consider the adhesive strength between the two bodies to be a constant, thereby 
neglecting ageing or oxidation mechanisms. Naturally, the approaches detailed in this paper can be developed further to include the presence of lubricants, ageing, and contrast between material properties as in abrasive wear, but this will be the topic of future work.

Section 2 describes our model atomistic pair potential. We discuss how this potential may be interpreted as a coarse-grained potential or discrete-element potential [12-14], in which case a discrete point should be interpreted as a group of atoms or grains, giving access to larger physical sizes. Section 3 summarizes the main components of our atomistic model for adhesive wear. One may distinguish between three fundamental asperity-level mechanisms behind wear: atom-by-atom attrition in the light load limit [15-17], gradual smoothing by dislocation plasticity [18-21] and amorphization [22], as well as fracture-induced third body formation [23-25]. The chosen geometry imposes a large overlap between opposing asperities and/or large loads, and aims to explore the transition between plastic smoothing of asperities and debris formation. We do not investigate the atom-by-atom attrition mechanism in this paper. In Section 4, we show that our potential is able to reproduce the ductile to brittle transition of contacting asperities [26]. The transition occurs at a critical junction size that depends on material properties. Contact junctions above the critical length scale form a debris particle, while smaller junctions deform plastically, and may form a debris particle at a later stage of the sliding history if the junction size then reaches the critical length scale. This gives a deterministic evaluation at the debris level of the wear coefficient in Archard's wear law. $K$ is either 1 (for large junctions) or 0 (for small junctions). Remarkably, it is not a probability anymore. Section 5 explores the life of a debris particle. We restrict our attention to contact junctions that are large enough to generate a debris particle and examine if Archard's law is recovered at the asperity level. We summarize the findings of a recent paper [27] in which we demonstrate that in the presence of strong adhesive forces, the frictional work is a good predictor of wear volume at the single asperity level, instead of the normal force component as used in Archard's wear law. Section 6 investigates the question of interactions, in the process of debris formation, between contact asperities. For nearby contact junctions, we show that crack shielding mechanisms occur, resulting in larger debris sizes. We argue that these mechanisms might be key to explain the transition from mild to severe wear [28]. Finally, Section 7 explores the upscaling of these findings in a BEM mesoscale model consisting of an elastic rough surface under normal loading. We examine the microcontact maps and obtain a direct measure of the wear coefficient for Archard's wear law [29].

\section{A simple model atomistic potential}

Previous atomistic modeling studies of adhesive wear predict a continual smoothing of surface asperities rather than the formation of debris particles $[8,9,22$, 30-33], inconsistent with macroscopic experimental observations. This can be understood in light of the challenge of scales discussed earlier. Simulation sizes amenable to reasonable computation times on modern computers are orders of magnitude smaller than the process zone sizes needed for generating wear particles by nucleation and propagation of subsurface cracks in many materials. These process zone sizes can be estimated by the square of the ratio of fracture toughness to yield strength. For instance, it is of the order of mm or above for metals, orders of magnitude above world-record atomistic simulations.

This long-standing quest for physics-based simulations of wear debris formation has been recently addressed through a coarse-grained atomistic approach [26]. This permits the simultaneous description of plastic deformation and fracture phenomena at a desirable length scale. In this approach, the material is modeled as a set of discrete points. System responses beyond the elastic limit (i.e., plasticity and fracture) are controlled by interactions between those points. For instance, the brittle/ductile response of the system, which is controlled through the competition between surface energy and the energy associated with plastic slip, can be tuned by modifying the potential well, the shape of potential tail and cut-off radius.

Considering a nearest-neighbor pairwise potential, we modified the long range character of the Morse potential [34] without disturbing the short range interactions (elastic properties) as follows: 


$$
\frac{V(r)}{\varepsilon}= \begin{cases}\left(1-e^{-\alpha\left(r-r_{o}\right)}\right)^{2}-1, & r<1.1 r_{o} \\ c_{1} \frac{r^{3}}{6}+c_{2} \frac{r^{2}}{2}+c_{3} r+c_{4}, & 1.1 r_{o} \leq r \leq r_{\mathrm{cut}} \\ 0, & r_{\mathrm{cut}} \leq r\end{cases}
$$

where the $r_{\text {cut }}$ parameter defines the potential cut-off radius and controls the interaction length scale and $c_{1}$ to $c_{4}$ are parameters. A smaller $r_{\text {cut }}$ makes the tail of the potential steeper, thereby increasing the yield strength. $r_{o}$ is the equilibrium bond distance at $T=0$ and $\varepsilon$ is the bond energy. The 1.1 factor ensures constant elastic properties up to $10 \%$ strain. This allows us to study the influence of inelastic properties while keeping the elastic properties fixed. In a coarse-grained interpretation of this potential [26], the distance between discrete points determines the scale at which plasticity and fracture phenomena are simulated. Interpreting this distance as the spacing between atoms gives classical MD results, in which plasticity and fracture are refined to a single dislocation and the rupture of an atomic bond respectively.

Figure 1(a) shows the bond energy versus atomic bond length for six different cut-off radii, with the smallest (P6 potential) corresponding to the most brittle behavior. In order to obtain the shear strength associated with each potential, we performed 2D indentation simulations. To model non-adhesive contact, we only considered the pure repulsive contribution of the interfacial potential. The indentation responses of all potentials are shown in Fig. 1(b). To extract the hardness value from these curves, we plot the contact pressure as the ratio of the indentation force $(P)$ to the projected contact area $(A)$, versus indentation depth [35]. Surface atoms within the cut-off radius of the interfacial potential are considered to define the atomic area of contact [36-38] Accordingly, the projected contact area at the atomic scale is computed [39]. Upon continued loading, the stress remains at a relatively constant value, which we take as the hardness. The critical shear strength $\tau$ corresponding to each potential is estimated as $\tau=\frac{1}{3 \sqrt{3}} H$ [40]. In simulations with full interfacial adhesion the junction strength $\sigma_{j}$ is taken equal to $\tau$. Note that in a 2D setup, plastic deformation occurs on three in-plane dislocation slip planes. We also performed 3D indentation simulations to characterize 3D coarse-grained potentials using a spherical indenter, see Fig. 1.
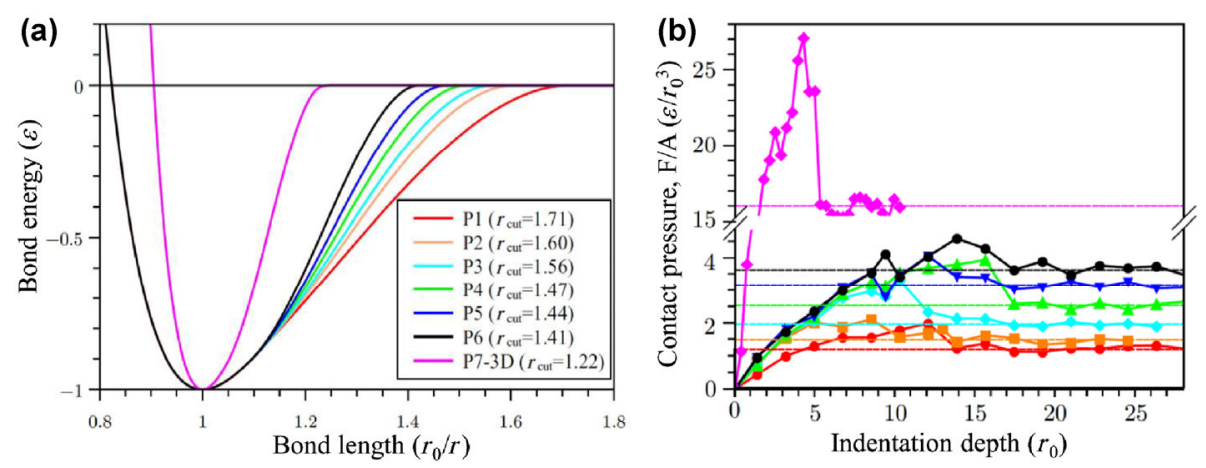

(c)
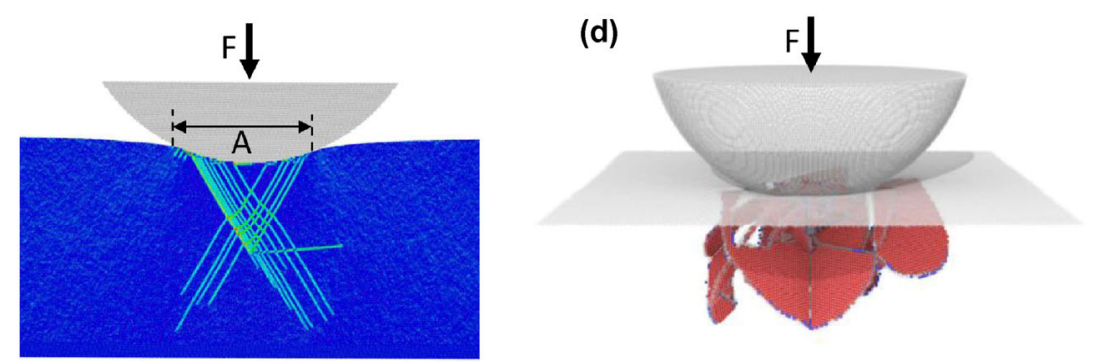

Fig. 1 (a) The bond energy versus atomic bond length is plotted for 2D (P1-P6) and 3D (P7) coarse-grained potentials. Bond energy and length are normalized by the depth of the potential well $(\varepsilon)$ and the equilibrium bond length $\left(r_{0}\right)$, respectively. (b) Contact pressure versus indentation depth obtained from $2 \mathrm{D}$ and $3 \mathrm{D}$ indentation simulations. The contact pressure is computed as the indentation force divided by the projected contact area. The plateau values are considered to be the effective hardness. (c) and (d) show snapshots of indentation simulations, showing plastic activity under the indenter in 2D (with P6 potential) and in 3D (with P7 potential). 


\section{A simple model for adhesive wear at the asperity level}

Besides the different parametrizations described in the previous section, the simulations performed using LAMMPS [41] differ in initial geometry, loading and boundary conditions (Fig. 2). These conditions are described in this section, where all quantities are expressed in reduced units as we model a single atom type with fundamental quantities $m, r_{0}, \varepsilon$, and $k_{B}$, which are respectively the atom mass, the equilibrium bond length, the equilibrium bond energy, and the Boltzmann constant.

To reduce the computational resources needed, most of the simulations are run in $2 \mathrm{D}$, with some $3 \mathrm{D}$ calculations run on high-performance computing platforms. The model consists of two contacting solids with a focus on the atomistic mechanisms at the contacting asperities: to this end, the simulation box size is large enough along the vertical direction to diminish boundary effects, while periodic boundary conditions are applied along the horizontal direction. The top body slides continuously at a constant velocity $v=0.001-0.05 \sqrt{\varepsilon m^{-1}}$, which is applied at the top atoms. The bottom atoms of the bottom body are instead fixed horizontally. Both bodies can translate vertically, so that the simulation box can expand or shrink upon asperities collision. To keep the bodies in contact, a constant vertical force $f_{y}$ of magnitude up to $0.02 \varepsilon r_{0}^{-1}$ per atom is applied on the top and bottom boundaries. A temperature of $0.1 \varepsilon k_{B}^{-1}$ is enforced by means of Langevin thermostats with a damping parameter of $0.05 r_{0} / \sqrt{\varepsilon m^{-1}}$ applied on two layers of atoms on each body (see Fig. 2). The simulation box has horizontal size $l_{x}=200-600 r_{0}$ and vertical size $l_{y}=$ $400-1000 r_{0}$. Surfaces are atomistically flat except for the presence of asperities. Different cases have been analysed including a single asperity (Fig. 2(a)), interlocking asperities leading to one contact junction (Fig. 2(b)), and a pair of contact junctions separated by a distance $\lambda$ (Fig. 2(c)). Within the set of simulations conducted in Ref. [26], we varied the asperity size $\left(d=20-140 r_{0}\right)$ and shape (semicircular, half sine, rectangular, triangular). The integration time step for the velocity Verlet algorithm is $0.0025 r_{0} / \sqrt{\varepsilon m^{-1}}$.

\section{Critical length scale for ductile to brittle transition}

Our simple model potential allows us to examine the asperity-level origins of adhesive wear mechanisms. As detailed in the previous section, a large number of adhesive wear simulations with different geometrical configurations, boundary conditions, and bulk and surface properties were conducted. These simulations demonstrated two adhesive wear mechanisms: gradual
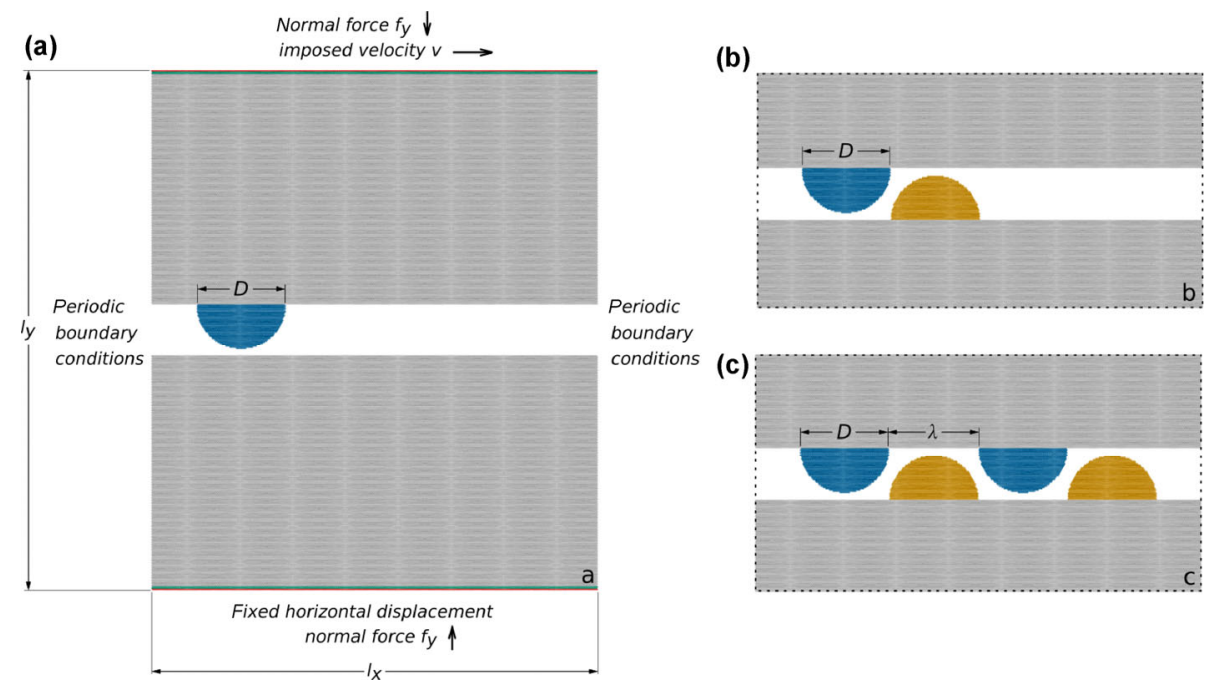

Fig. 2 Schematic for the atomistic simulations. (a) Boundary conditions and single-asperity surface topography. In green and red are the layers of atoms where the thermostats and displacements are applied respectively. (b) Interlocking asperities surface topography, with one asperity on each surface. (c) Interlocking asperities surface topography, with two asperities spaced at a distance $\lambda$ on each surface. 
asperity smoothing by plastic deformation (see Fig. 3(a)) versus fracture-induced debris formation (Fig. 3(b)). Examining a range of simulation parameters, we found that the size of the asperity contact junction and the strength of the adhesive bond dictate the adhesive wear mechanism. Ultimately, hard/brittle materials with large asperities and strong adhesive bonding at the asperity contact junctions favour the debris formation mechanism over the asperity smoothing mechanism.

Inspired by previous theoretical insights $[42,43]$ and this new understanding [26], we find that the transition between plastic smoothing and fracture-induced debris mechanisms can be predicted with a simple analytic model. The model considers that the transition from plastic deformation to debris formation occurs when the stored elastic energy is large enough to create new wear-debris particle surfaces. The maximum stored elastic energy depends on the junction shear strength and the junction size $d$, and the surface energy determines the energy cost of creating a debris particle. This yields the existence of a critical length scale $d^{*}$. Asperity junctions larger than $d^{*}$ produce wear debris by fracture while smaller ones smooth out plastically. The analytic model gives

$$
d^{*}=c \cdot \frac{\Delta w}{\left(\sigma_{j}^{2} / G\right)}
$$

where $G$ is the shear modulus and $\sigma_{j}$ is the shear strength of the junction. $\Delta w$ is the energy associated to newly created free surfaces (i.e., decohesion energy). $c$ is a shape factor combining contributions of all geometrical factors (e.g., asperities shape and junction configuration). For the removal of an idealized 2D circular and 3D spherical debris, we obtain $c=\frac{8}{\pi}$ and $c=3$ respectively. This model can be also understood in terms of a crack growth model [44, 45], in which the detailed kinetics of crack growth and other dissipative mechanisms (e.g., plasticity) could be taken into account. Figure 3 illustrates this ductile to brittle transition at a critical junction size in the context of a $3 \mathrm{D}$ asperity [27]. In case of a junction size smaller than the critical size, Fig. 3(a), the strong adhesive forces yield a severe plastic deformation of the asperities. This process has been routinely observed in the literature with classical atomistic potentials (see for instance Ref. [30]). With repeated sliding of the simulation box size, one observes a continuous smoothing of the asperity. If the lateral dimension of the simulation box is smaller than $d^{*}$, this process will lead eventually to a full contact between the two blocks. However, if the simulation box size and junction size are larger than the critical length scale, as in Fig. 3(b), a debris particle is formed.

The proposed simple analytic model can explain and predict the operative mechanisms in AFM wear experiments, where the AFM tip has been reported to wear due to adhesive forces via both gradual smoothing [17-19, 20, 21, 24, 46, 47] and the creation of fracture induced debris $[24,25,48]$.
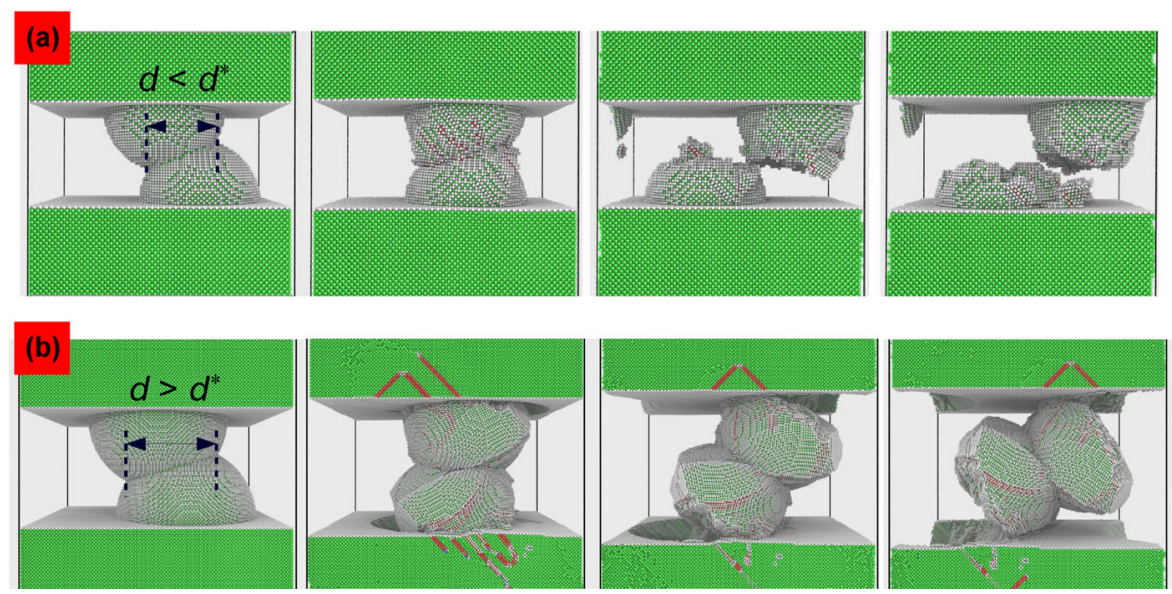

Fig. 3 Numerical simulations distinguish two wear mechanisms at the asperity level. (a) shows the plastic smoothing mechanism in the absence of wear debris particle for a small asperities junction. (b) presents the fracture-induced particle formation mechanism for a large asperity junction. See [26, 27] for detailed information about the simulations. 


\section{Quantitative prediction of debris size}

\subsection{Birth of a debris particle}

A direct modeling of wear debris formation [27] enables quantifying the amount of detached material at the onset of debris formation and studying the origins of long-standing macroscopic wear observations: (i) the wear volume (i.e., total volume of wear debris) is independent of apparent area of contact $[49,50]$, (ii) within a certain range of applied load, the wear rate (i.e., wear volume per sliding distance) is linearly proportional to the macroscopic load acting normal to the interface, like in Archard's wear law [49, 3], and (iii) the wear volume is proportional to the frictional work (i.e., the product of frictional force and sliding distance) [5, 51,52].

A recent systematic set of adhesive wear simulations [27] showed universal features of the process of debris formation despite the variety of parameters and configurations examined. Initially, a strong adhesive bond (junction) forms between contacting asperities. Subsequent sliding leads to the junction growth by localized inelastic deformation, until crack nucleation and growth ensues at the two corners of the junction loaded in tension. These simulations reveal three main observations about the process of single debris particle formation:

- The diameter of the debris particle upon formation is the same as the maximum junction size, independent of the parameters and configurations of a particular simulation. This observation rationalizes the correlation between the real contact area and the volume of the debris particle, which is a central tenet of Archard's wear model [3].

- The debris volume does not correlate with the product of applied normal load and sliding distance (i.e., Archard's wear relation). This observation is in fact not surprising for an isolated debris particle considering that two opposing asperities can collide, adhere, and form a debris particle even in the absence of an applied normal force. In this situation, the contact area of a single asperity junction is largely affected by the asperity's geometry $[53,36]$, shear loading [38,54] and adhesion [55]. Therefore, the normal applied force does not linearly depend on the junction size and, as a result, cannot predict debris volume. This finding suggests that the macroscopically observed linear relation between the wear volume and the normal force may be reconstructed via multiasperity contact models, where the contact is a load-controlled process [56].

- Alternatively it is found [27] that the debris volume scales with the work of tangential force that is carried by the junction, with a proportionality constant of 1 over the junction shear strength. This can be explained by the fact that the tangential force transmitted across the asperity junction provides an indication of the maximum asperity junction area. Therefore, as the asperity junction size controls the debris size, the wear debris volume can be quantified via the work done by the tangential component of the load carried by the junction.

These debris-level wear observations [27] together with the concept of the critical junction size [26] confirm that the debris formation is a deterministic process at the asperity level (i.e., wear coefficient of unity at the single debris level if the junction size is above the critical size). It will guide us in proposing a first-level estimation of the wear coefficient in Archard's law. For this, one needs to statistically analyze the junction size probability in a multiasperity contact setting [29], as detailed later in Section 7.

\subsection{Life of a debris particle}

Once a debris particle has been formed, its following evolution affects both the wear volume and the changes in the surfaces roughness. At the end of an experimental wear test, the shapes of the debris particles can be investigated and are found to be related to the mechanism of wear involved [57]. The particles produced during a wear process where adhesion is relevant are characterized by an elliptic shape [5], and their elongation is small when compared with particles produced in wear processes of the abrasive type [57]. In our 2D simulations of adhesive wear, the debris particle that forms displays a circular shape most of the most of the time, with very little elongation. Of course, 3D simulations would be more relevant to investigate shape evolution.

Preliminary simulations of 2D debris conducted over long time scales reveal that the formed debris particle volume increases with time, although slowly 
and at a decreasing rate. Therefore the wear is characterized by first a high wear rate at the onset of debris formation and a transition to a slower wear rate, which is consistent with experimental observations [58]. Future work will present a continuum mechanics model to explain why the asymmetry in the loading favours crack propagation within the bulk at the trailing edge. For circular debris shapes we have observed that the basic mechanism for debris growth is detachment of matter from the substrate, and attachment to the debris. In addition, we have also observed that the debris size tends to saturate with time. More work is needed to understand in details the wear particle growth rate. In particular several mechanisms could break this growth pattern. The debris particle could develop a concave shape with time, leading to stress concentrations within the debris particle. Also the particle could break/fragment upon the action of compressive forces, a process that would be favoured by creating weak layers in the debris particle owing to ageing. We do not currently consider ageing in our simulations, which is an artefact differentiating our results from real wear experiments. Accounting for these mechanisms could eventually lead to the death of the debris particle. Another fate would be its evacuation from the contact interface.

\section{Interactions between microcontacts}

Until now, we considered the problem of wear on the level of a single debris particle. This is clearly an approximation, which may fail in several common cases: debris particles will come into contact with each other during the wear process, debris particles will interact with asperities, and microcontacts cannot necessarily be separated into individual asperities that behave independently.

Here, we consider the latter case. At low normal load, we expect the contacts to be spaced out. Moreover, many contacts will have sizes $d<d^{*}$ and will not form debris particles; a model of the macroscopical consequences of this observation will be discussed in the next section. For the moment, we look at the case of high normal load, where more contacts have sizes $d>d^{*}$ and the average distance between the contact spots shrinks. It is known from literature that the contact solutions for closely-spaced contact junctions are different from individual junctions $[59,60]$. As detailed in Section 4, the detachment of a debris particle is the result of a subsurface crack, which will be influenced by the modified stress field. By extending the simulation setup from Section 4 from a single junction to two junctions, we were able to investigate this in more detail in a recent work [28]. By bringing the contacting asperities close together, the detachment mechanism switches: single asperity mechanisms (either plastic smoothing or debris formation, Figs. 4(a)-4(b)) are replaced by collective deep subsurface cracks that lead to the detachment of a single, large debris particle (Fig. 4(c)). By systematically varying the junction size $d$ and the spacing $\lambda$ between the junctions, we found that-in this specific case-the transition from individual debris formation to a combined mechanism occurs for $\lambda<d$. Furthermore, the volume of the resulting debris particle is no longer related to the size of the individual junctions.

This latter observation has implications for the "bottom-up" prediction of wear coefficients. When the debris particle size scales with the junction size [27], we should recover a proportionality between the normal load and the wear rate as for example in Archard's wear law $[5,3,49,50]$ : the actual contact area, i.e., the sum of the junction sizes, is a function of normal load [61] and thus the resulting debris volume is, too. In the case of interacting junctions, on the other hand, the wear rate is no longer related to the actual contact area, but to some effective contact area that is somewhere between the actual contact area and the apparent macroscopic contact area. This mirrors the transition from mild to severe wear, for which a sudden increase of the wear coefficient at a critical load has been observed [62-71]. While the current investigations only treat a simplified model case, they give a first possible explanation for this transition. Further modeling and experiments are needed to quantify the phenomenon.

It remains to be explained why the junctions interact at all instead of being worn off individually. For this, we first look at the initial stages of the wear process for far-spaced junctions in Fig. 5(a). We can see that the asperities come into contact and tensile stress concentrations build up. With increasing sliding distance, 

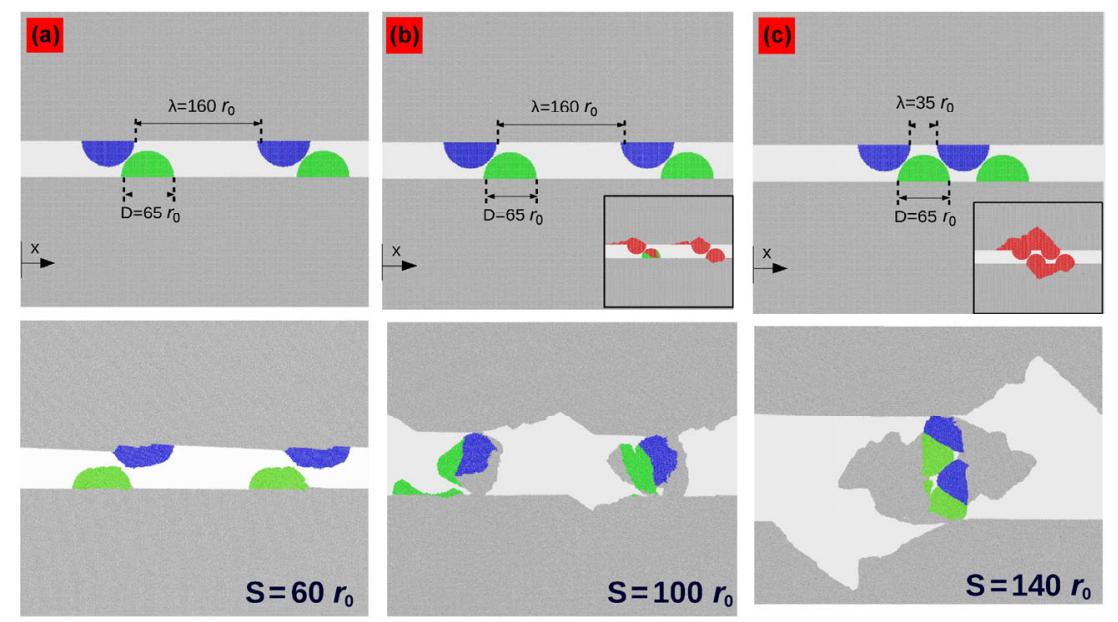

Fig. 4 (a) Asperity smoothing without debris formation, where the asperity junctions are smaller than the critical junction size. This is ensured by reducing the interfacial adhesion, which establishes a weak interfacial junction between interacting asperities and consequently a very large critical junction size [26]. (b) Debris formation at the asperity level in the presence of a high interfacial adhesion, where strong junctions are established. In this case, debris particles of the same size as the asperity junctions are formed individually. (c) Once asperity pairs get close to each other, they interact through their underneath inelastic stress fields, which results in the formation of a large debris particle. In this condition, no correlation between the debris size and junction size is observed. For a better visualization of detached volume, debris atoms are highlighted in the initial configuration in the insets of Figs. 4(b) and 4(c).
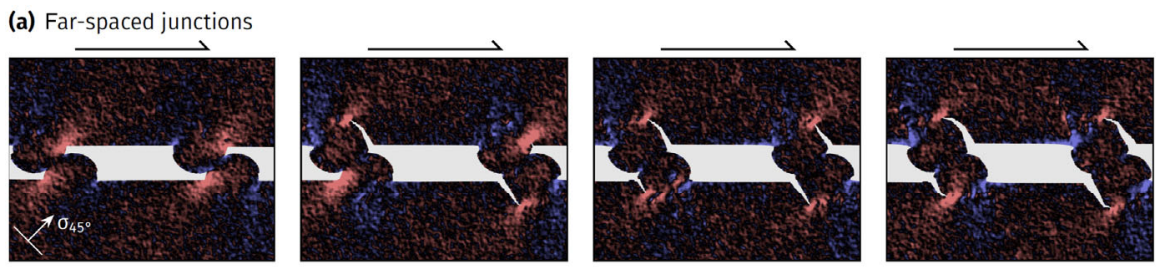

(b) Closely-spaced junctions
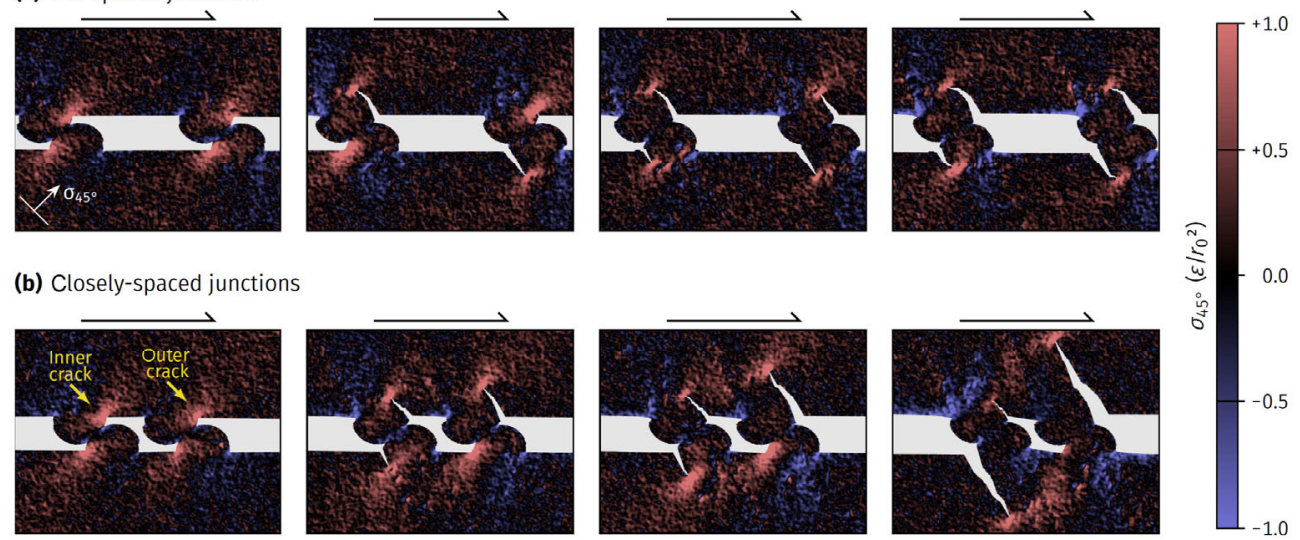

Fig. 5 Mechanisms of debris formation. Sliding simulations are shown, the sliding direction is indicated by the black arrows. The color coding shows the stress component $\sigma_{45^{\circ}}$, which acts perpendicular to the crack tips and is responsible for the crack nucleation and propagation.

these stress concentrations lead to the successive formation of cracks that cause the detachment of two debris particles. It is immediately visible that their volume is close to the volume of the asperities. If the asperities are closer, Fig. 5(b), the initial steps are very similar. Stress concentrations build up in the same way and it even seems that cracks occur at the same places. Further sliding, though, changes the picture. The "outer crack" at the leading junction (see label in the figure) grows and starts to unload the "inner crack" at the trailing junction, which then closes. This mechanism is dictated by the geometry of the problem: due to the imposed sliding direction, a sufficiently propagated outer crack can "envelope" the trailing asperity and completely unload it, as shown in Fig. 5(a), but not vice versa. In the textbook case [72], parallel crack shielding becomes effective roughly when the distance between the two cracks is less than twice their length. Assuming that the depth of a crack at non-interacting junctions is roughly the asperity size $d$ [27], the distance $\lambda$ must be approximately equal to or smaller than $d$ to reach this distance between the 
inner and outer crack. This can justify the observations above. Additionally, the modified subsurface stress field seems to always lead to a deeper propagation of the cracks.

While the model is very simple and a quantitative picture clearly needs to take more realistic 3D geometries into account, it already gives us insight into the complexity of asperity interactions. In fact, given the varied contact geometries that occur when rough surfaces touch, it raises the question of how to exactly define an asperity. Do we need to account for every "internal valley" or should we prefer a more coarse-grained view?

\section{A mesoscale model for wear}

Finally, we present two mesoscale wear models that up-scale the relations derived at the asperity level from atomistic insights to a multi-asperity setting [29]. These models allow the estimation of the total wear rate as a function of the interface physical properties, the surfaces' geometrical properties and the normal load applied on the system.

\subsection{Archard's wear model}

In his seminal work [3], Archard puts forth a model for macroscopic wear that is composed of two fundamental parts: a single-asperity wear model and a multi-asperity contact model. At the single-asperity level, Archard makes the following considerations:

- given two hemi-spherical asperities in contact in a zone of radius $a$ (which is half the contact junction size $d$ ), the resulting wear particle has a volume proportional to $a^{3}$,

- the sliding distance necessary to produce the particle is $2 a$,

- each asperity encounter has a probability $K$ of forming a wear particle.

Archard uses these hypotheses in conjunction with his multi-asperity contact model to derive, in the case of a rigid-plastic material, the well known ArchardHolm equation:

$$
R(W)=K \frac{W}{H}
$$

where $R$ is the wear rate, $W$ is the applied normal load and $H$ is the flow stress of the material. This equation has been widely used, but unfortunately Archard does not provide a way to quantify $K$, so applications of this equation exclusively rely on parameter fitting with experimental data. Archard and Hirst [62] make the claim that "one of the most important problems in an understanding of wear is to explain the magnitude of the probability of the production of a wear particle at an asperity encounter".

However, the last of Archard's hypotheses introduces $K$ to account for the fact that not all asperity encounters form wear particles. It is clear from Ref. [26] that the particle formation process follows a Griffith-like criterion and is a deterministic event at the asperity level, so $K$ cannot be introduced at the asperity level. We now present a definition of $K$ at the mesoscale.

Consider a rough surface contact situation (Fig. 6) where the true contact area is composed of a multitude of smaller contacts, here called contact clusters. Since the surface height distribution is random, the distribution of the area of the contact clusters is also random. We denote $A$ the random variable representing the area of a single contact cluster. This variable follows the probability density function $p(A, W)$, which depends on the load.

We suppose that the wear particle formation process is governed by a critical contact area $A^{*}$ derived from the previously discussed critical length scale $d^{*}$. A wear particle is therefore formed if and only if $A>A^{*}$. Since $K$ is the probability that an asperity encounter forms a debris particle, we obtain the natural definition [29]

$$
K \equiv P\left(A>A^{*}, W\right)=\int_{A^{*}}^{\infty} p(A, W) d A
$$

Experimental evidence [62] and our previous simulation results show that the wear rate is proportional to the real contact area in the mild wear regime, with a proportionality constant called the "wear coefficient". In Archard's model, the wear coefficient is $K$, the probability of wear particle formation. However, this interpretation of the wear coefficient is not physically justified by Archard.

An alternative interpretation of the wear coefficient not relying on Archard's interpretation is possible [29]. Consider a system of finite size, so that there are $N(W)$ contact clusters for a given load. We define the un-normalized cluster size distribution as $n(A, W)=$ 
$N(W) \cdot p(A, W)$ and the asperity level wear rate $R_{1}(A)=\omega A$, with $\omega$ a shape factor equal to $1 / 3$ in the case of hemi-spherical asperities (the wear rate is the ratio of volume of wear debris over sliding distance, which in the simple case of hemi-spherical asperities gives a $1 / 3$ factor.). These are used in the computation of the total wear rate

$$
\begin{aligned}
R(W) & =\int_{A^{*}}^{\infty} R_{1}(A) n(A, W) d A \\
& =\int_{A^{*}}^{\infty} \omega A n(A, W) d A=\omega A_{c}^{*}(W)
\end{aligned}
$$

where $A_{c}^{*}$ is the cumulative area of contact clusters forming wear particles. If we now introduce $\mathcal{K}(W)=$ $\frac{A_{c}^{*}(W)}{A_{c}(W)}$, we can write the total wear rate as $R(W)=$ $\mathcal{K}(W) \omega A_{c}(W) . \mathcal{K}$ is then the wear coefficient, and naturally arises from the up-scaling process of single asperity wear rate, which was discussed in the previous sections, to a multi-asperity setting. The central difference with Archard's purely probabilistic interpretation is that this second interpretation of the wear coefficient emerges from the sum of volumes of debris particles, which depend on local contact junction areas.

The results of Fig. 6 summarize several BEM calculations in which we have normally loaded elastic self-affine rough surfaces, with $\lambda_{s}$ being the shortest wave length in the surface spectrum. Figure 6 shows that the wear coefficient $K$ according to Archard's interpretation reproduces the experimentally $[49,62]$ observed behavior of mild wear. Specifically, $K$ reaches a plateau implying that there exists a range of loads for which the wear coefficient is independent of load. The wear coefficient introduced in Ref. [29] does not reproduce this behavior, but this is likely due to the constitutive model of the solids in contact, as they are here considered linear elastic, whereas in reality plastic deformations are expected. Another important limitation of this direct interpretation of the wear coefficient is that it does not take into account inelastic interactions between asperities (e.g., crack shielding, third-body contact, plasticity). These were highlighted in a simple 2D context in Section 6. Interactions between contact clusters are certainly even richer in a 3D setting, in which non-convex nearby contact patches may interact through elastic forces and crack shielding

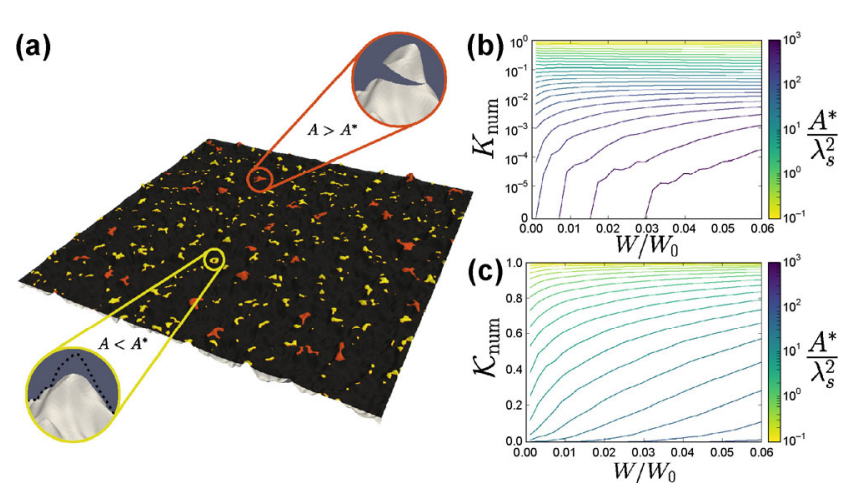

Fig. 6 (a) Multi-asperity contact setting with a fractal rough surface [29]. Yellow clusters are smaller than $A^{*}$ and deform plastically upon sliding. Red clusters are larger than $A^{*}$. (b) shows $K$, the probability of wear particle formation according to Archard's interpretation. We see that for large values of $A^{*}, K$ is initially zero, then increases with the load up to a plateau. This is a behavior observed in experiments [49, 62]. (c) shows the wear coefficient according to our model that does not rely on Archard's assumption. In this figure, the wear coefficient also transitions from zero, but increases up to one. This is likely due to the contact model that only considers a purely elastic material.

mechanisms. The question of how to merge these contact patches in an effective contact area $A$, and in essence of what is an asperity, is far from trivial and is completely neglected in this first attempt. Despite the current limitations of our model, we highlight that mesoscale continuum models provide a remarkably efficient approach to obtain physics-based estimate of wear coefficients. Future models should aim at accounting for plastic deformations, interactions between microcontacts, surface roughness evolution, transport of particles and eventual reattachment or evacuation from the contact interface.

\section{Conclusion}

We have synthesized our recent theoretical advances towards physics-based modeling of adhesive wear. These advances were made possible by the use of a model atomic potential. Atomic simulations revealed a ductile to brittle transition for large enough contact junction sizes. Small junctions deform plastically, whereas large junctions break to form debris which is transported along the sliding contact interface. The critical length scale at which this transition occurs is a function of the material properties. The junction size also determines the debris particle size at its birth. After 
birth, under our idealized condition of full adhesion, the debris particle will continue to grow but at a decreasing rate. We have also shown that interactions between nearby contact junctions are due to crack shielding mechanisms. These interactions become increasingly important at large loads and may explain the change from the mild to the severe wear regime. Finally, we have incorporated some of these findings into a continuum model in order to obtain a deterministic estimation of the wear coefficient used in Archard's wear law.

Clearly, some of the research efforts that were described are still in their infancy. Tribological mechanisms being extremely complex, we make no claims at pretending to present a complete picture of adhesive wear, and in fact we are still a long way from reaching this stage. Perhaps the most pressing efforts should be toward including more materials science in our model. More realistic atomistic potentials should be used. Ageing and alteration of adhesive properties should be accounted for as these are likely to change the long term growth of debris particles. Because these more accurate potentials will come at a larger computational cost than our model potential, the simulations should be conducted on high-performance computing platforms. The influence of sliding velocity and temperature should also be investigated, as well as interactions between contact junctions in a 3D setting. Finally, a lot of room exists for improving the continuum models that we have presented here.

Nonetheless, despite all the current limitations, we emphasize the importance of anchoring tribology research to the fundamentals to make long term progress. Tribology is at a turning point. Current simulation and experimental capabilities give us unprecedented means to revisit empirical wear models with a fresh scientifically-grounded look.

Open Access: The articles published in this journal are distributed under the terms of the Creative Commons Attribution 4.0 International License (http:// creativecommons.org/licenses/by/4.0/), which permits unrestricted use, distribution, and reproduction in any medium, provided you give appropriate credit to the original author(s) and the source, provide a link to the Creative Commons license, and indicate if changes were made.

\section{References}

[1] Meng H C, Ludema K C. Wear models and predictive equations: their form and content. Wear 181-183: 443-457 (1995)

[2] Hatchett C, ESQ F R S. IV. Experiments and observations on the various alloys, on the specific gravity, and on the comparative wear of gold. Being the substance of a report made to the Right Honourable the Lords of the Committee of Privy Council, appointed to take into consideration the state of the coins of this Kingdom, and the present establishment and constitution of his Majesty's Mint. Phil Trans $R$ Soc Lond 93: 43-194 (1803)

[3] Archard J F. Contact and rubbing of flat surfaces. J Appl Phys 24(8): 981-988 (1953)

[4] Schirmeisen A. Wear: One atom after the other. Nat Nanotechnol 8(2): 81-82 (2013)

[5] Rabinowicz E. Friction and Wear of Materials. New York (USA): Wiley, 1995.

[6] Ewen J P, Gattinoni C, Thakkar F, Morgan N, Spikes H A, Dini D. Nonequilibrium molecular dynamics investigation of the reduction in friction and wear by carbon nanoparticles between iron surfaces. Tribol Lett 63(3): 38 (2016)

[7] Onodera T, Martin J M, Minfray C, Dassenoy F, Miyamoto A. Antiwear chemistry of ZDDP: coupling classical md and tight-binding quantum chemical md methods (TB-QCMD). Tribol Lett 50(1): 31-39 (2013)

[8] Stoyanov P, Romero P A, Merz R, Kopnarski M, Stricker M, Stemmer P, Dienwiebel M, Moseler M. Nanoscale sliding friction phenomena at the interface of diamond-like carbon and tungsten. Acta Mater 67: 395-408 (2014)

[9] Pastewka L, Moser S, Gumbsch P, Moseler M. Anisotropic mechanical amorphization drives wear in diamond. Nat Mater 10(1): 34-38 (2011)

[10] Sha Z, Branicio P, Pei Q X, Sorkin V, Zhang Y W. A modified tersoff potential for pure and hydrogenated diamondlike carbon. Comput Mater Sci 67: 146-150 (2013)

[11] Hu X L, Altoe M V P, Martini A. Amorphization-assisted nanoscale wear during the running-in process. Wear 370-371: 46-50 (2017)

[12] Cundall P A, Strack O D L. A discrete numerical model for granular assemblies. Géotechnique 29(1): 47-65 (1979)

[13] Renouf M, Massi F, Fillot N, Saulot A. Numerical tribology of a dry contact. Tribol Int 44(7-8): 834-844 (2011)

[14] Fillot N, Iordanoff I, Berthier Y. Wear modeling and the third body concept. Wear 262(7-8): 949-957 (2007)

[15] Jacobs T D B, Gotsmann B, Lantz M A, Carpick R W. On the application of transition state theory to atomic-scale wear. Tribol Lett 39(3): 257-271 (2010) 
[16] Bhaskaran H, Gotsmann B, Sebastian A, Drechsler U, Lantz M A, Despont M, Jaroenapibal P, Carpick R W, Chen Y, Sridharan K. Ultralow nanoscale wear through atom-byatom attrition in silicon-containing diamond-like carbon. Nat Nanotechnol 5(3): 181-185 (2010)

[17] Gotsmann B, Lantz M A. Atomistic wear in a single asperity sliding contact. Phys Rev Lett 101(12): 125501 (2008)

[18] Sato T, Ishida T, Jalabert L, Fujita H. Real-time transmission electron microscope observation of nanofriction at a single ag asperity. Nanotechnology 23(50): 505701 (2012)

[19] Merkle A P, Marks L D. Liquid-like tribology of gold studied by in situ TEM. Wear 265(11-12): 1864-1869 (2008)

[20] Vahdat V, Grierson D S, Turner K T, Carpick R W. Mechanics of interaction and atomic-scale wear of amplitude modulation atomic force microscopy probes. ACS Nano 7(4): 3221-3235 (2013)

[21] Chung K H. Wear characteristics of atomic force microscopy tips: A reivew. Int J Precis Eng Manuf 15(10): 2219-2230 (2014)

[22] De Barros Bouchet M I, Matta C, Vacher B, Le-Mogne T, Martin J M, von Lautz J, Ma T, Pastewka L, Otschik J, Gumbsch P, Moseler M. Energy filtering transmission electron microscopy and atomistic simulations of tribo-induced hybridization change of nanocrystalline diamond coating. Carbon 87: 317-329 (2015)

[23] Stoyanov P, Romero P A, Järvi T T, Pastewka L, Scherge M, Stemmer P, Fischer A, Dienwiebel M, Moseler M. Experimental and numerical atomistic investigation of the third body formation process in dry tungsten/tungsten-carbide tribo couples. Tribol Lett 50(1): 67-80 (2013)

[24] Liu J J, Notbohm J K, Carpick R W, Turner K T. Method for characterizing nanoscale wear of atomic force microscope tips. ACS Nano 4(7): 3763-3772 (2010)

[25] Chung K H, Kim D E. Fundamental investigation of micro wear rate using an atomic force microscope. Tribol Lett 15(2): 135-144 (2003)

[26] Aghababaei R, Warner D H, Molinari J F. Critical length scale controls adhesive wear mechanisms. Nat Commun 7: 11816 (2016)

[27] Aghababaei R, Warner D H, Molinari J F. On the debrislevel origins of adhesive wear. Proc Natl Acad Sci USA 114(30): 7935-7940 (2017)

[28] Aghababaei R, Brink T, Molinari J F. Asperity-level origins of transition from mild to severe wear. Phys Rev Lett 120(18): 186105 (2018)

[29] Frérot L, Aghababaei R, Molinari J F. A mechanistic understanding of the wear coefficient: From single to multiple asperities contact. J Mech Phys Solids 114: 172-184 (2018)
[30] Spijker P, Anciaux G, Molinari J F. Dry sliding contact between rough surfaces at the atomistic scale. Tribol Lett 44(2): 279-285 (2011)

[31] Sørensen M R, Jacobsen K W, Stoltze P. Simulations of atomic-scale sliding friction. Phys Rev B 53(4): 2101-2113 (1996)

[32] Zhong J, Shakiba R, Adams J B. Molecular dynamics simulation of severe adhesive wear on a rough aluminum substrate. J Phys D: Appl Phys 46(5): 055307 (2013)

[33] Sha Z D, Sorkin V, Branicio P S, Pei Q X, Zhang Y W, Srolovitz D J. Large-scale molecular dynamics simulations of wear in diamond-like carbon at the nanoscale. Appl Phy Lett 103(7): 073118 (2013)

[34] Morse P M. Diatomic molecules according to the wave mechanics. II. vibrational levels. Phys. Rev. 34(1): 57-64 (1929)

[35] Oliver W C, Pharr G M. An improved technique for determining hardness and elastic modulus using load and displacement sensing indentation experiments. $J$ Mater Res 7(6): 1564-1583 (1992)

[36] Luan B Q, Robbins M O. The breakdown of continuum models for mechanical contacts. Nature 435(7044): 929-932 (2005)

[37] Mo Y F, Turner K T, Szlufarska I. Friction laws at the nanoscale. Nature 457(7233): 1116-1119 (2009)

[38] Mo Y F, Szlufarska I. Roughness picture of friction in dry nanoscale contacts. Phys Rev B 81(3): 035405 (2010)

[39] Ziegenhain G, Urbassek H M, Hartmaier A. Inuence of crystal anisotropy on elastic deformation and onset of plasticity in nanoindentation: A simulational study. J Appl Phys 107(6): 061807 (2010)

[40] Ashby M F, Jones D R H. Engineering Materials. Oxford (UK): Pergamon, 1980.

[41] Plimpton S. Fast parallel algorithms for short-range molecular dynamics. J Comput Phys 117(1): 1-9 (1995)

[42] Rabinowicz E. Influence of surface energy on friction and wear phenomena. J Appl Phys 32(8): 1440-1444 (1961)

[43] Rabinowicz E. The effect of size on the looseness of wear fragments. Wear 2(1): 4-8 (1958)

[44] Rice J R. Dislocation nucleation from a crack tip: An analysis based on the Peierls concept. J Mech Phys Solids 40(2): 239-271 (1992)

[45] Griffith A A. VI. The phenomena of rupture and flow in solids. Philos Trans R Soc A Math Phys Eng Sci 221(582592): 163-198 (1921)

[46] Tao Z, Bhushan B. Surface modification of AFM silicon probes for adhesion and wear reduction. Tribol Lett 21(1): $1-16$ (2006) 
[47] Jacobs T D B, Carpick R W. Nanoscale wear as a stressassisted chemical reaction. Nat Nanotechnol 8(2): 108-112 (2013)

[48] Bhushan B, Sundararajan S. Micro/nanoscale friction and wear mechanisms of thin films using atomic force and friction force microscopy. Acta Mater 46(11): 3793-3804 (1998)

[49] Burwell J T, Strang C D. On the empirical law of adhesive wear. J Appl Phys 23(1): 18-28 (1952)

[50] Holm R. Frictional wear in metallic contacts without current. In Electric Contacts. Holm R, Ed. Berlin, Heidelberg (Germany): Springer, 1946: 232-242.

[51] Uetz H, Föhl J. Wear as an energy transformation process. Wear 49(2): 253-264 (1978)

[52] Reye T. Zur theorie der zapfenreibung. J Der Civilingenieur 4: 235-255 (1860)

[53] Popov V L, Pohrt R, Li Q. Strength of adhesive contacts: Influence of contact geometry and material gradients. Friction 5(3): 308-325 (2017)

[54] McFarlane J S, Tabor D. Adhesion of solids and the effect of surface films. Proc R Soc A Math Phys Eng Sci 202(1069): 224-243 (1950)

[55] Pastewka L, Robbins M O. Contact between rough surfaces and a criterion for macroscopic adhesion. Proc Natl Acad Sci USA 111(9): 3298-3303 (2014)

[56] Persson B N J. Elastoplastic contact between randomly rough surfaces. Phys Rev Lett 87(11): 116101 (2001)

[57] Stachowiak G P, Stachowiak G W, Podsiadlo P. Automated classification of wear particles based on their surface texture and shape features. Tribol Int 41(1): 34-43 (2008)

[58] Queener C A, Smith T C, Mitchell W L. Transient wear of machine parts. Wear 8(5): 391-400 (1965)

[59] Komvopoulos K, Choi D H. Elastic finite element analysis of multi-asperity contacts. J Tribol 114(4): 823-831 (1992)

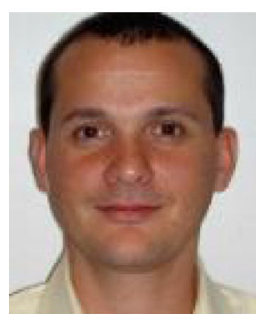

Jean-François MOLINARI. He is the director of the Computational Solid Mechanics Laboratory (LSMS) at the École Polytechnique Fédérale of Lausanne (EPFL), Switzerland. He graduated in 1997 with a MS degree in mechanical engineering from the University of Technology of Compiègne,
[60] Caroli C, Nozières P. Hysteresis and elastic interactions of microasperities in dry friction. Eur Phys J B 4(2): 233-246 (1998)

[61] Bowden F P, Tabor D. The area of contact between stationary and between moving surfaces. Proc R Soc A Math Phys Eng Sci 169(938): 391-413 (1939)

[62] Archard J F, Hirst W. The wear of metals under unlubricated conditions. Proc R Soc A Math Phys Eng Sci 236(1206): $397-410$ (1956)

[63] Lim S C, Ashby M F. Overview no. 55 wear-mechanism maps. Acta Metall 35(1): 1-24 (1987)

[64] Kitsunai H, Kato K, Hokkirigawa K, Inoue H. The transitions between microscopic wear modes during repeated sliding friction observed by a scanning electron microscope tribosystem. Wear 135(2): 237-249 (1990)

[65] Hokkirigawa K. Wear mode map of ceramics. Wear 151(2): 219-228 (1991)

[66] Wang Y S, Hsu S M. Wear and wear transition modeling of ceramics. Wear 195(1-2): 35-46 (1996)

[67] Adachi K, Kato K, Chen N. Wear map of ceramics. Wear 203-204: 291-301 (1997)

[68] Zhang J, Alpas A. Transition between mild and severe wear in aluminium alloys. Acta Mater 45(2): 513-528 (1997)

[69] Kato K, Adachi K. Wear of advanced ceramics. Wear 253(11-12): 1097-1104 (2002)

[70] Hsu S M, Shen M. Wear prediction of ceramics. Wear 256(9-10): 867-878 (2004)

[71] Wang S Q, Wang L, Zhao Y T, Sun Y, Yang Z R. Mild-tosevere wear transition and transition region of oxidative wear in steels. Wear 306(1-2): 311-320 (2013)

[72] Murakami Y. Stress Intensity Factors Handbook. New York (USA): Pergamon Press, 1987.

France. He also obtained a MS degree in aeronautics in 1997 from the California Institute of Technology, USA, and graduated with a PhD degree from Caltech in 2001. He then held professor positions at the Johns Hopkins University, USA, and ENS Cachan, France, before joining EPFL. His research interests cover multiscale modeling with applications to fracture mechanics and tribology. 


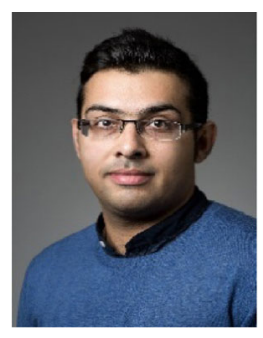

Ramin AGHABABAEI. He obtained his $\mathrm{PhD}$ degree in mechanical engineering from National University of Singapore (NUS) in 2012. He joined the Computational Solid Mechanics Laboratory (LSMS) at École Polytechnique Fédérale of Lausanne (EPFL) as a post-doctoral fellow. In September 2017, he joined the Engineering Department of Aarhus

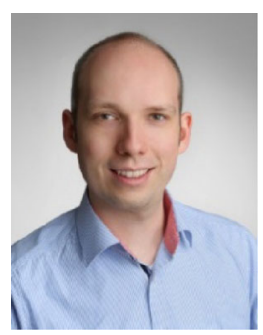

Tobias BRINK. He received his doctoral degree in materials science from the Technische Universität Darmstadt, Germany. He is currently working at the Computational Solid Mechanics Laboratory (LSMS) at the École Polytechnique Fédérale

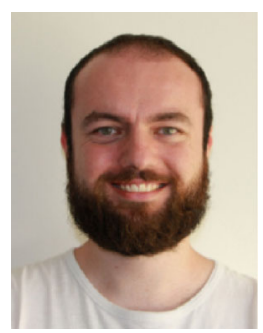

Lucas FRÉROT. He holds a bachelor of science and a master of science in civil engineering from the École Polytechnique Fédérale de Lausanne (EPFL), with a focus on computational methods in the modeling

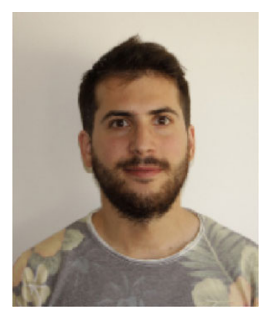

Enrico MILANESE. He trained as a civil engineer at the University of Padua, Italy, where he received both his bachelor and master degrees. He later investigated fracture avalanche behaviour in porous media at the
University in Denmark, as a tenure-track assistant professor, and the principle investigator of the surface mechanics group (SMG). The research interest of his group is to understand the underlying mechanics and physics of failure in materials surfaces at different scales with application to tribology and manufacturing.

of Lausanne (EPFL) in Switzerland on atomistic simulations of wear at the asperity level. In this context, his research interests include working towards understanding nano-scaled wear mechanisms and how they result in macroscopic wear relations.

of solids and structures. He joined the Computational Solid Mechanics Laboratory at EPFL in 2015 as a PhD student to conduct research on the elasto-plastic contact of solids with rough surfaces, with application to the study of friction and wear.

same university. Since 2016 he is pursuing a $\mathrm{PhD}$ at the Computational Solid Mechanics Laboratory at the École Polytechnique Fédérale of Lausanne (EPFL), Switzerland. His current research focusses on surface roughness and adhesive wear. 